\title{
Hadronization measurements in cold nuclear matter
}

\author{
Raphaël Dupré ${ }^{1}$ \\ ${ }^{1}$ Institut de Physique Nucléaire, CNRS/IN2P3 and Université Paris Sud, Orsay, France
}

\begin{abstract}
Hadronization is the non-perturbative process of QCD by which partons become hadrons. It has been studied at high energies through various processes, we focus here on the experiments of lepto-production of hadrons in cold nuclear matter. By studying the dependence of observables to the atomic number of the target, these experiments can give information on the dynamic of the hadronization at the femtometer scale. In particular, we will present preliminary results from JLab Hall B (CLAS collaboration), which give unprecedented statistical precision. Then, we will present results of a phenomenological study showing how HERMES data can be described with pure energy loss models.
\end{abstract}

\section{Introduction}

The hadronization of quarks has been studied through many reactions and has been found to be an universal process involving QCD at the perturbative and non perturbative levels. The study of $e^{+} e^{-}$and $e p$ collisions gives access to universal functions of fragmentation, $D_{i}^{h}(z)$, representing the probability for a quark of flavor $i$ to produce a hadron $h$ with a fraction $z$ of its momentum. In this article, we go besides this description and focus on the dynamic of the hadronization process, which can provide a new insights on hadronization beyond fragmentation functions.

We can decompose the process of hadronization in two parts (see figure 1), first, a part where the quark propagates and can be described with perturbative QCD; then, a part where it becomes a colorless prehadron expanding into a fully formed hadron. The latter phase is non perturbative and its end defines the formation time, while the former phase ends at the production time. The length of these times can be modeled based on various assumptions, but first principles calculations are not yet possible. We can however try to experimentally evaluate these times, by comparing fragmentation functions obtained from experiments on different nuclear targets [1].

To characterize hadronization in nuclei, we use the multiplicity ratio $R_{A}^{h}$ and the transverse momentum broadening $\Delta P_{T}^{2} . R_{A}^{h}$ is directly linked to the attenuation of the hadrons in the medium, given by $1-R_{A}^{h}$, and is defined as

$$
R_{A}^{h}=\frac{N_{A}^{h} / N_{A}^{e}}{N_{D}^{h} / N_{D}^{e}},
$$

with $N_{A}^{h}$ the number of hadrons $h$ produced on a target $A$ and $N_{A}^{e}$ the number of electrons scattered from the target $A, D$ being for the deuterium target which usually serves as a reference. The transverse momentum broadening is defined by

$$
\Delta P_{T}^{2}=\left\langle p_{T}^{2}\right\rangle_{A}-\left\langle p_{T}^{2}\right\rangle_{D}
$$




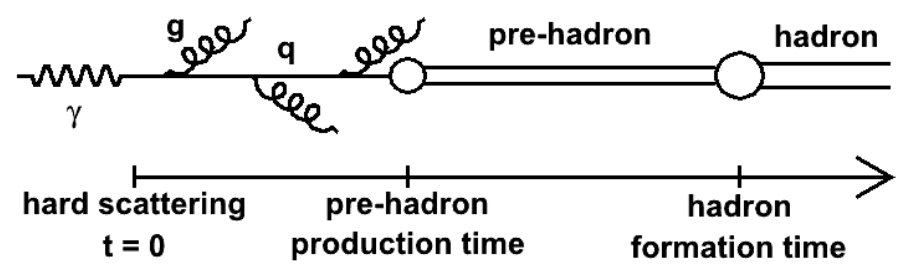

Figure 1. The hadronization of a quark from Deep Inelastic Scattering (DIS) illustrating the production and formation time concepts.

with $\left\langle p_{T}^{2}\right\rangle_{A}$ the mean transverse momentum of hadrons produced in the target $A$. We present these observables as functions of the usual kinematic factors for semi-inclusive DIS: $Q^{2}=-q^{2}$, the 4momentum squared of the virtual photon; $v=p \cdot q / \sqrt{p^{2}}$, the energy of the virtual photon in the target rest frame (with $p$ the 4-momentum of the target nucleon); $z=p_{h} \cdot p / q \cdot p$, the fractional energy carried by the hadron (with $p_{h}$ the 4-momentum of the produced hadron) and finally $p_{T}^{2}$ the transverse momentum squared of the hadron, the virtual photon defining the longitudinal direction.

\section{Past Measurements}

The measurement of nuclear Deep Inelastic Scattering (DIS) at SLAC in 1978 [2] was a pioneer and provided the first clear information on hadron production on nuclei by using a $20.5 \mathrm{GeV}$ electron beam. A reduction of the hadron production rate with larger nuclear masses was observed, indicating that the nuclear material absorbed a part of the hadron spectrum. This attenuation was also observed to be stronger at higher momentum fraction of the hadrons $(z)$, but no dependence was observed on the other kinematical variables.

The European Muon Collaboration (EMC) confirmed these results in 1991 [3] at higher energy (up to $280 \mathrm{GeV}$ muon beam energy). Moreover, the combination of SLAC and EMC data showed that the absorption was reduced at high $v$. The EMC also observed a fast increase of the multiplicity ratio at high $p_{T}^{2}$. This effect is similar to the Cronin effect from heavy ion collisions [4], which is believed to be generated by multiple soft scattering.

The E665 experiment [5], carried at the Fermi National Accelerator Laboratory (FNAL), also provided interesting data. Their study showed the importance of the target fragmentation region and its dominance at intermediate rapidity and subsequently the importance to select properly the current fragmentation in future experiments. They also measured slow protons numbers in the target region, which appeared to give information on the impact parameter of the interaction.

The HERMES collaboration published a large set of data [6,7] using the $27.5 \mathrm{GeV}$ positron beam in DESY. Their results gave new insight on hadronization because of their precision and the identification of individual particles $\left(\pi^{+}, \pi^{0}, \pi^{-}, K^{+}, K^{-}, p\right.$ and $\left.\bar{p}\right)$. The measurement of the three pions independently gave similar results, which were also compatible with the previous experiments measuring all hadrons together. The multiplicity ratio of $\pi^{+}$shows an increase with $v$ commonly explained by the boost of the produced hadrons, a decrease with $z$ that can be linked to reduced production time at higher $z$ [8] and a sharp increase as function of $p_{T}^{2}$ caused by multiple soft scatterings. The HERMES collaboration also provided, for the first time, results for the $\Delta P_{T}^{2}$ observable, offering a new insight into the effects of the nuclear medium. Indeed, this observable is believed to give a direct access to the parton energy loss features $[8,9]$. 


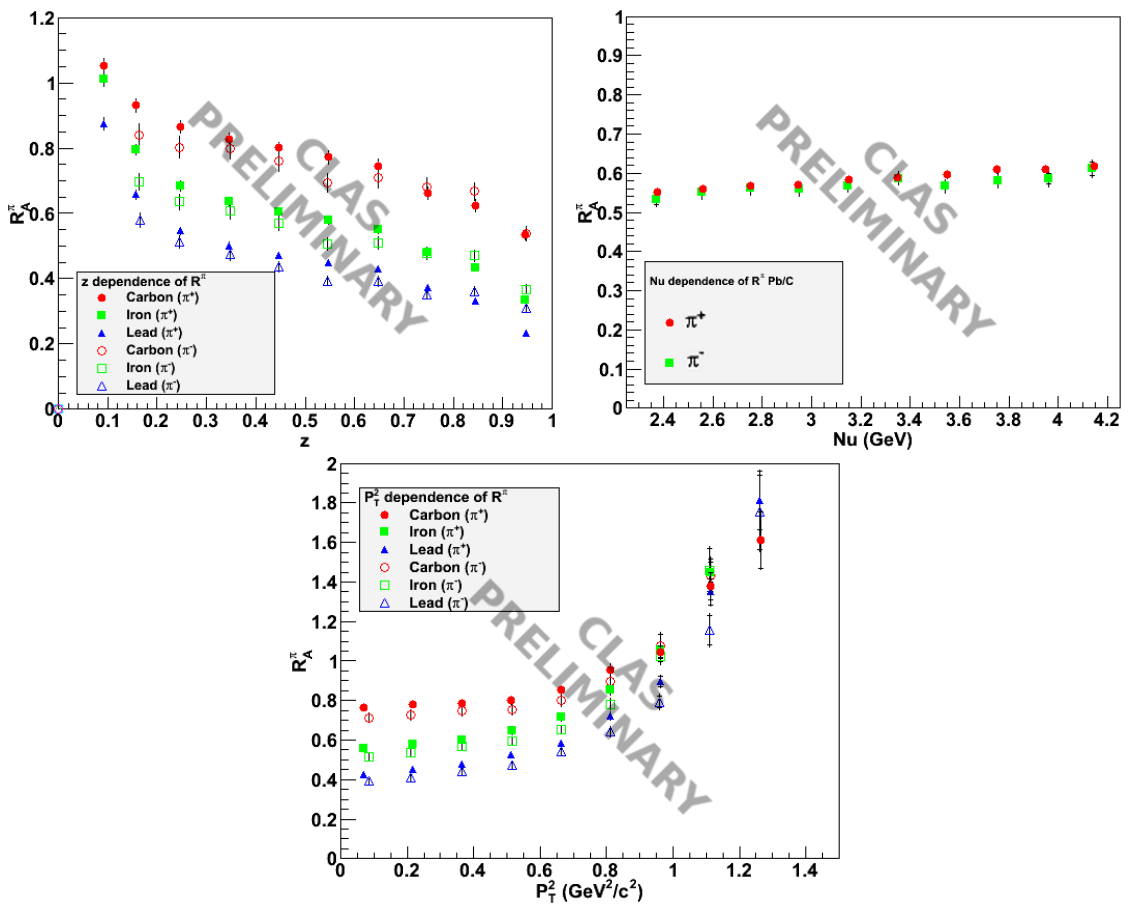

Figure 2. CLAS preliminary multiplicity ratios as function of $z, v$ and $p_{T}^{2}$ for charged pions ${ }^{1}$.

In conclusion, HERMES provided the highest precision data on hadronization in cold nuclear matter ( $\mathrm{He}, \mathrm{Ne}, \mathrm{Kr}$ and $\mathrm{Xe}$ ) offering a clear view of the field and confirming important hypothesis on $\Delta P_{T}^{2}$, but also raising new questions on attenuation mechanisms for heavier hadrons production, kaons and protons in particular [6].

\section{The CLAS Experiment}

The CLAS experiment at JLab provides data at lower energy (5 GeV electron beam) but with large statistics and a large set of nuclei. We first see in figure 2 that we reproduce all the previous results for the multiplicity ratio, i.e. a decrease with $z$, an increase with $v$ and a strong increase with $p_{T}^{2}$.

The target used for the experiment: ${ }^{2} \mathrm{H}, \mathrm{C}, \mathrm{Al}, \mathrm{Fe}, \mathrm{Sn}$ and $\mathrm{Pb}$ give a wider range in $A$ than any previous experiments, allowing CLAS to explore the $A$ dependence of our observables in more details. Figure 3 shows both $R_{A}^{h}$ and $\Delta P_{T}^{2}$ as function of $A^{1 / 3}$, which is directly proportional to the length of medium encountered by the parton during hadronization. We observe that while the nuclear effects increase with nuclear size, they are not doing so linearly. This is an unexpected result that will need careful modeling and in particular constrain the production length at this energy.

The large statistics gathered by the CLAS collaboration also allows to explore the transverse momentum broadening effects. In figure 4, we show $\Delta P_{T}^{2}$ as function of $z$ and $Q^{2}$, the latter being shown in a small $v$ range. This kind of constrain allows to isolate QCD effects varying with $Q^{2}$ from simple

\footnotetext{
${ }^{1}$ The multiplicity ratio as function of $v$ is calculated with carbon as reference instead of deuterium. For design reasons, this reduces systematic errors and helps to clarify the small slope observed in this case.
} 

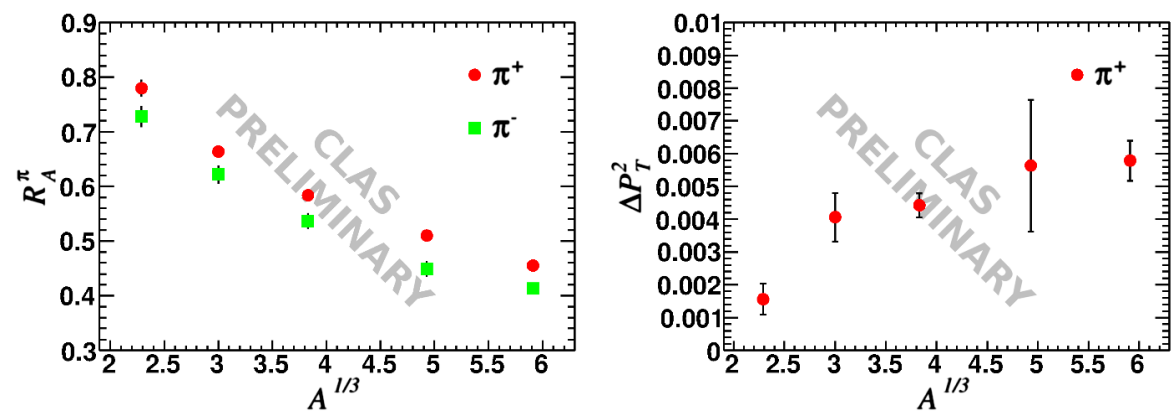

Figure 3. CLAS multiplicity ratio and transverse momentum broadening as function of $A^{1 / 3}$ for charged pions.

Lorentz boost and is only possible because of the very large body of data available to CLAS. The result as function of $z$ is strongly increasing and does not make a plateau like in HERMES. We can attribute this strange feature to the effect of Fermi motion, which is stronger at the low energy of CLAS than in HERMES [10]. However, the rather flat result for the $Q^{2}$-dependence is unlikely to be affected by Fermi motion and is surprising since models such as [9] predict an increase that was hinted by HERMES results [7].
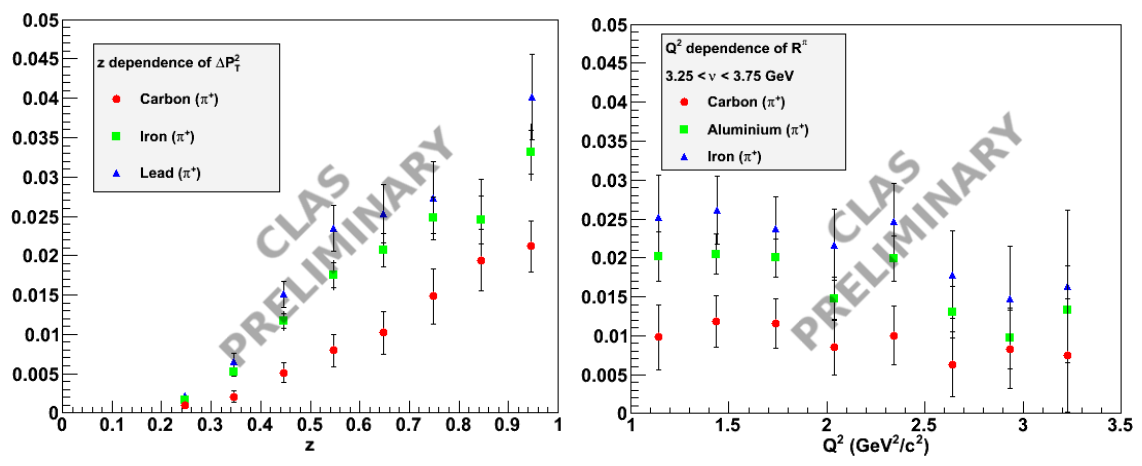

Figure 4. CLAS multiplicity transverse momentum broadening as function of $z$ and $Q^{2}$ for positive pions.

The CLAS data show many other features [11, 12], which provide very interesting inputs for theoretical models because the large statistics available allow for stringent selection cuts and multidimensional analysis.

\section{Parton Energy Loss Models}

To interpret the data, the models for the interactions in nuclear medium are of very different nature. The quark propagating in nuclear matter can be described within perturbative QCD similarly to the quarks in hot nuclear matter [13]. This treatment allows also to characterize the medium and, in particular, its gluonic component [13, 14]. Some models consider the energy loss effect to be small and focus on the behavior of the prehadron. It is possible, in this case, to use diffractive diquark production in order to calibrate the process [8] or one can use the hadron cross sections as a base. 
However, in the latter case, the direct use of hadronic cross sections fails to describe the data [15] and it should be adapted. For example, in [16], the cross section is null before the production time and then increases linearly to reach its full hadronic cross section at the formation time.

There have been difficulties for pure parton energy loss models - i.e. models including only parton energy loss and no hadronic interaction - to describe both the multiplicity ratio and the transverse momentum broadening from HERMES. In these models, the two are linked by the transport coefficient of the medium $\hat{q}$, which is the transverse momentum squared acquired by the parton per unit of distance. This parameter serves in parton energy loss models both to set the absorption and the transverse momentum broadening. However, values for $\hat{q}$ necessary to explain the observed absorption have been generally found to be too large to match the observed $\Delta P_{T}^{2}$. These models generally use the simple assumption that $\hat{q}=p_{T}^{2} / z^{2} / L$, we checked this hypothesis using a parton energy loss calculation with a full simulation of the fragmentation. We found that the link between $\hat{q}$ and $\Delta P_{T}^{2}$ is much more complex [10] than though before. In figure 5, we can see our implementation of the calculation in PYTHIA, taking into account the effect of the absorption on $\Delta P_{T}^{2}$, describing both observables at the same time with a $\hat{q}=0.36 \mathrm{GeV}^{2} / \mathrm{fm}$. The main difference with the previous approach is that the full Monte-Carlo simulation takes into account the correlation between absorption and long path in the nuclear medium. Indeed, the partons that go through the longest paths in the nuclear medium are the least likely to produce hadrons and therefore to contribute to the $\Delta P_{T}^{2}$ measurement. Our result shows that taking this bias into account is necessary to describe HERMES data completely.
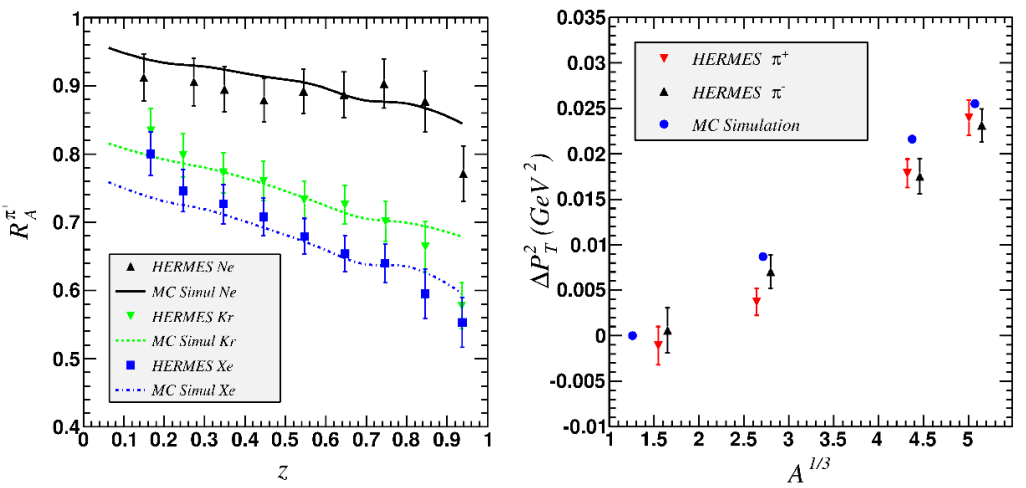

Figure 5. On the left, multiplicity ratio from HERMES compared with our pure energy loss model [10]. On the right, the transverse momentum broadening as function of $A^{1 / 3}$ using $\hat{q}=0.35 \mathrm{GeV}^{2} / \mathrm{fm}$ found by matching the simulated ratio to data.

\section{Conclusions}

The recent data of high precision from the HERMES and CLAS collaborations allow to better constrain theoretical models and to give a new insight into physical quantities like the production and formation time or the transport coefficient of the medium. We also show that the correlation between absorption and transverse momentum broadening is not negligible and needs careful implementation in models.

Future measurements are already planned to progress even further in these directions. At JLab, a new experiment is already approved to run after the upgrade of the beam to $12 \mathrm{GeV}$ using the new 
CLAS12 spectrometer. The aim of this experiment will be to pass a new level in precision and allow the extraction of the production and formation times. There will be also a focus on kaon and baryon hadronization that cannot be explored in detail with current CLAS data.

\section{References}

[1] A. Accardi, F. Arleo, W.K. Brooks, D. D’Enterria, V. Muccifora, Riv. Nuovo Cim. 32, 439 (2010), 0907. 3534

[2] L. Osborne, C. Bolon, R. Lanza, D. Luckey, D. Roth et al., Phys.Rev.Lett. 40, 1624 (1978)

[3] J. Ashman et al. (European Muon Collaboration), Z.Phys. C52, 1 (1991)

[4] A. Accardi (2002), hep-ph/0212148

[5] M. Adams et al. (E665 Collaboration), Z.Phys. C65, 225 (1995)

[6] A. Airapetian et al. (HERMES Collaboration), Nucl.Phys. B780, 1 (2007), 0704 . 3270

[7] A. Airapetian et al. (HERMES Collaboration), Phys.Lett. B684, 114 (2010), 0906.2478

[8] B. Kopeliovich, J. Nemchik, E. Predazzi, A. Hayashigaki, Nucl.Phys. A740, 211 (2004), hep-ph/0311220

[9] S. Domdey, D. Grunewald, B. Kopeliovich, H. Pirner, Nucl.Phys. A825, 200 (2009), 0812 . 2838

[10] A. Accardi, R. Dupre, To be published (2015)

[11] W. Brooks, H. Hakobyan, Nucl.Phys. A830, 361C (2009), 0907. 4606

[12] T. Mineeva, PhD dissertation (2013)

[13] R. Baier, Y.L. Dokshitzer, A.H. Mueller, S. Peigne, D. Schiff, Nucl. Phys. B484, 265 (1997), hep-ph/9608322

[14] B. Kopeliovich, I. Potashnikova, I. Schmidt, Phys.Rev. C81, 035204 (2010), 1001.4281

[15] M. Gyulassy, M. Plumer, Nucl.Phys. B346, 1 (1990)

[16] K. Gallmeister, U. Mosel, Nucl.Phys. A801, 68 (2008), nucl-th/0701064 